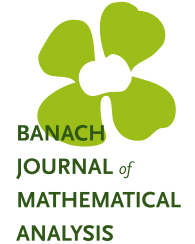

Banach J. Math. Anal. 10 (2016), no. 4, 727-749

http://dx.doi.org/10.1215/17358787-3649986

ISSN: $1735-8787$ (electronic)

http://projecteuclid.org/bjma

ANALYSIS

\title{
POISSON SEMIGROUP, AREA FUNCTION, AND THE CHARACTERIZATION OF HARDY SPACE ASSOCIATED TO DEGENERATE SCHRÖDINGER OPERATORS
}

\author{
JIZHENG HUANG, ${ }^{1}$ PENGTAO LI, ${ }^{2^{*}}$ and YU LIU ${ }^{3}$ \\ Communicated by M. A. Ragusa
}

Abstract. Let

$$
L f(x)=-\frac{1}{\omega(x)} \sum_{i, j} \partial_{i}\left(a_{i j}(\cdot) \partial_{j} f\right)(x)+V(x) f(x)
$$

be the degenerate Schrödinger operator, where $\omega$ is a weight from the Muckenhoupt class $A_{2}$ and $V$ is a nonnegative potential that belongs to a certain reverse Hölder class with respect to the measure $\omega(x) d x$. Based on some smoothness estimates of the Poisson semigroup $e^{-t \sqrt{L}}$, we introduce the area function $S_{P}^{L}$ associated with $e^{-t \sqrt{L}}$ to characterize the Hardy space associated with $L$.

\section{INTRODUCTION}

As a special class of Calderón-Zygmund singular integrals, the area function is a useful way of building bridges between real analysis and complex analysis. In harmonic analysis, the area function is an important tool to characterize the function spaces. In [7], Fefferman and Stein proved that the area function and the nontangential maximal function are equivalent in the sense of $L^{p}\left(\mathbb{R}^{n}\right)$; they also established the area function characterization of the Hardy spaces $H^{p}\left(\mathbb{R}^{n}\right)$. From then on, the Hardy space was extended to other settings. (We refer the reader to [3], [2], [8], [18], [10], and the references therein, which investigate more general

Copyright 2016 by the Tusi Mathematical Research Group.

Received Nov. 6, 2015; Accepted Jan. 6, 2016.

${ }^{*}$ Corresponding author.

2010 Mathematics Subject Classification. Primary 42B30; Secondary 35J10, 42B25.

Keywords. Hardy space, Schrödinger operator, atom, area integral, Poisson semigroup, area function. 


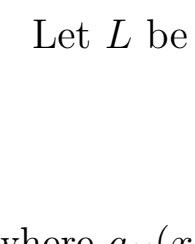

Banach J. Math. Anal. 10 (2016), no. 4, 727-749

http://dx.doi.org/10.1215/17358787-3649986

ISSN: $1735-8787$ (electronic)

http://projecteuclid.org/bjma

ANALYSIS

\title{
POISSON SEMIGROUP, AREA FUNCTION, AND THE CHARACTERIZATION OF HARDY SPACE ASSOCIATED TO DEGENERATE SCHRÖDINGER OPERATORS
}

\author{
JIZHENG HUANG, ${ }^{1}$ PENGTAO LI, ${ }^{2^{*}}$ and YU LIU ${ }^{3}$ \\ Communicated by M. A. Ragusa
}

Abstract. Let

$$
L f(x)=-\frac{1}{\omega(x)} \sum_{i, j} \partial_{i}\left(a_{i j}(\cdot) \partial_{j} f\right)(x)+V(x) f(x)
$$

be the degenerate Schrödinger operator, where $\omega$ is a weight from the Muckenhoupt class $A_{2}$ and $V$ is a nonnegative potential that belongs to a certain reverse Hölder class with respect to the measure $\omega(x) d x$. Based on some smoothness estimates of the Poisson semigroup $e^{-t \sqrt{L}}$, we introduce the area function $S_{P}^{L}$ associated with $e^{-t \sqrt{L}}$ to characterize the Hardy space associated with $L$.

\section{INTRODUCTION}

As a special class of Calderón-Zygmund singular integrals, the area function is a useful way of building bridges between real analysis and complex analysis. In harmonic analysis, the area function is an important tool to characterize the function spaces. In [7], Fefferman and Stein proved that the area function and the nontangential maximal function are equivalent in the sense of $L^{p}\left(\mathbb{R}^{n}\right)$; they also established the area function characterization of the Hardy spaces $H^{p}\left(\mathbb{R}^{n}\right)$. From then on, the Hardy space was extended to other settings. (We refer the reader to [3], [2], [8], [18], [10], and the references therein, which investigate more general

Copyright 2016 by the Tusi Mathematical Research Group.

Received Nov. 6, 2015; Accepted Jan. 6, 2016.

${ }^{*}$ Corresponding author.

2010 Mathematics Subject Classification. Primary 42B30; Secondary 35J10, 42B25.

Keywords. Hardy space, Schrödinger operator, atom, area integral, Poisson semigroup, area function. 


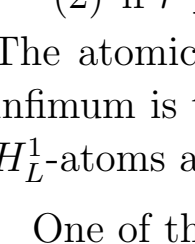

Banach J. Math. Anal. 10 (2016), no. 4, 727-749

http://dx.doi.org/10.1215/17358787-3649986

ISSN: $1735-8787$ (electronic)

http://projecteuclid.org/bjma

ANALYSIS

\title{
POISSON SEMIGROUP, AREA FUNCTION, AND THE CHARACTERIZATION OF HARDY SPACE ASSOCIATED TO DEGENERATE SCHRÖDINGER OPERATORS
}

\author{
JIZHENG HUANG, ${ }^{1}$ PENGTAO LI, ${ }^{2^{*}}$ and YU LIU ${ }^{3}$ \\ Communicated by M. A. Ragusa
}

Abstract. Let

$$
L f(x)=-\frac{1}{\omega(x)} \sum_{i, j} \partial_{i}\left(a_{i j}(\cdot) \partial_{j} f\right)(x)+V(x) f(x)
$$

be the degenerate Schrödinger operator, where $\omega$ is a weight from the Muckenhoupt class $A_{2}$ and $V$ is a nonnegative potential that belongs to a certain reverse Hölder class with respect to the measure $\omega(x) d x$. Based on some smoothness estimates of the Poisson semigroup $e^{-t \sqrt{L}}$, we introduce the area function $S_{P}^{L}$ associated with $e^{-t \sqrt{L}}$ to characterize the Hardy space associated with $L$.

\section{INTRODUCTION}

As a special class of Calderón-Zygmund singular integrals, the area function is a useful way of building bridges between real analysis and complex analysis. In harmonic analysis, the area function is an important tool to characterize the function spaces. In [7], Fefferman and Stein proved that the area function and the nontangential maximal function are equivalent in the sense of $L^{p}\left(\mathbb{R}^{n}\right)$; they also established the area function characterization of the Hardy spaces $H^{p}\left(\mathbb{R}^{n}\right)$. From then on, the Hardy space was extended to other settings. (We refer the reader to [3], [2], [8], [18], [10], and the references therein, which investigate more general

Copyright 2016 by the Tusi Mathematical Research Group.

Received Nov. 6, 2015; Accepted Jan. 6, 2016.

${ }^{*}$ Corresponding author.

2010 Mathematics Subject Classification. Primary 42B30; Secondary 35J10, 42B25.

Keywords. Hardy space, Schrödinger operator, atom, area integral, Poisson semigroup, area function. 


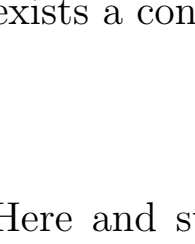

Banach J. Math. Anal. 10 (2016), no. 4, 727-749

http://dx.doi.org/10.1215/17358787-3649986

ISSN: $1735-8787$ (electronic)

http://projecteuclid.org/bjma

ANALYSIS

\title{
POISSON SEMIGROUP, AREA FUNCTION, AND THE CHARACTERIZATION OF HARDY SPACE ASSOCIATED TO DEGENERATE SCHRÖDINGER OPERATORS
}

\author{
JIZHENG HUANG, ${ }^{1}$ PENGTAO LI, ${ }^{2^{*}}$ and YU LIU ${ }^{3}$ \\ Communicated by M. A. Ragusa
}

Abstract. Let

$$
L f(x)=-\frac{1}{\omega(x)} \sum_{i, j} \partial_{i}\left(a_{i j}(\cdot) \partial_{j} f\right)(x)+V(x) f(x)
$$

be the degenerate Schrödinger operator, where $\omega$ is a weight from the Muckenhoupt class $A_{2}$ and $V$ is a nonnegative potential that belongs to a certain reverse Hölder class with respect to the measure $\omega(x) d x$. Based on some smoothness estimates of the Poisson semigroup $e^{-t \sqrt{L}}$, we introduce the area function $S_{P}^{L}$ associated with $e^{-t \sqrt{L}}$ to characterize the Hardy space associated with $L$.

\section{INTRODUCTION}

As a special class of Calderón-Zygmund singular integrals, the area function is a useful way of building bridges between real analysis and complex analysis. In harmonic analysis, the area function is an important tool to characterize the function spaces. In [7], Fefferman and Stein proved that the area function and the nontangential maximal function are equivalent in the sense of $L^{p}\left(\mathbb{R}^{n}\right)$; they also established the area function characterization of the Hardy spaces $H^{p}\left(\mathbb{R}^{n}\right)$. From then on, the Hardy space was extended to other settings. (We refer the reader to [3], [2], [8], [18], [10], and the references therein, which investigate more general

Copyright 2016 by the Tusi Mathematical Research Group.

Received Nov. 6, 2015; Accepted Jan. 6, 2016.

${ }^{*}$ Corresponding author.

2010 Mathematics Subject Classification. Primary 42B30; Secondary 35J10, 42B25.

Keywords. Hardy space, Schrödinger operator, atom, area integral, Poisson semigroup, area function. 


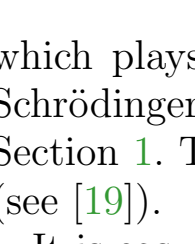

Banach J. Math. Anal. 10 (2016), no. 4, 727-749

http://dx.doi.org/10.1215/17358787-3649986

ISSN: $1735-8787$ (electronic)

http://projecteuclid.org/bjma

ANALYSIS

\title{
POISSON SEMIGROUP, AREA FUNCTION, AND THE CHARACTERIZATION OF HARDY SPACE ASSOCIATED TO DEGENERATE SCHRÖDINGER OPERATORS
}

\author{
JIZHENG HUANG, ${ }^{1}$ PENGTAO LI, ${ }^{2^{*}}$ and YU LIU ${ }^{3}$ \\ Communicated by M. A. Ragusa
}

Abstract. Let

$$
L f(x)=-\frac{1}{\omega(x)} \sum_{i, j} \partial_{i}\left(a_{i j}(\cdot) \partial_{j} f\right)(x)+V(x) f(x)
$$

be the degenerate Schrödinger operator, where $\omega$ is a weight from the Muckenhoupt class $A_{2}$ and $V$ is a nonnegative potential that belongs to a certain reverse Hölder class with respect to the measure $\omega(x) d x$. Based on some smoothness estimates of the Poisson semigroup $e^{-t \sqrt{L}}$, we introduce the area function $S_{P}^{L}$ associated with $e^{-t \sqrt{L}}$ to characterize the Hardy space associated with $L$.

\section{INTRODUCTION}

As a special class of Calderón-Zygmund singular integrals, the area function is a useful way of building bridges between real analysis and complex analysis. In harmonic analysis, the area function is an important tool to characterize the function spaces. In [7], Fefferman and Stein proved that the area function and the nontangential maximal function are equivalent in the sense of $L^{p}\left(\mathbb{R}^{n}\right)$; they also established the area function characterization of the Hardy spaces $H^{p}\left(\mathbb{R}^{n}\right)$. From then on, the Hardy space was extended to other settings. (We refer the reader to [3], [2], [8], [18], [10], and the references therein, which investigate more general

Copyright 2016 by the Tusi Mathematical Research Group.

Received Nov. 6, 2015; Accepted Jan. 6, 2016.

${ }^{*}$ Corresponding author.

2010 Mathematics Subject Classification. Primary 42B30; Secondary 35J10, 42B25.

Keywords. Hardy space, Schrödinger operator, atom, area integral, Poisson semigroup, area function. 


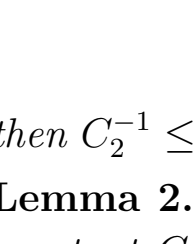

Banach J. Math. Anal. 10 (2016), no. 4, 727-749

http://dx.doi.org/10.1215/17358787-3649986

ISSN: $1735-8787$ (electronic)

http://projecteuclid.org/bjma

ANALYSIS

\title{
POISSON SEMIGROUP, AREA FUNCTION, AND THE CHARACTERIZATION OF HARDY SPACE ASSOCIATED TO DEGENERATE SCHRÖDINGER OPERATORS
}

\author{
JIZHENG HUANG, ${ }^{1}$ PENGTAO LI, ${ }^{2^{*}}$ and YU LIU ${ }^{3}$ \\ Communicated by M. A. Ragusa
}

Abstract. Let

$$
L f(x)=-\frac{1}{\omega(x)} \sum_{i, j} \partial_{i}\left(a_{i j}(\cdot) \partial_{j} f\right)(x)+V(x) f(x)
$$

be the degenerate Schrödinger operator, where $\omega$ is a weight from the Muckenhoupt class $A_{2}$ and $V$ is a nonnegative potential that belongs to a certain reverse Hölder class with respect to the measure $\omega(x) d x$. Based on some smoothness estimates of the Poisson semigroup $e^{-t \sqrt{L}}$, we introduce the area function $S_{P}^{L}$ associated with $e^{-t \sqrt{L}}$ to characterize the Hardy space associated with $L$.

\section{INTRODUCTION}

As a special class of Calderón-Zygmund singular integrals, the area function is a useful way of building bridges between real analysis and complex analysis. In harmonic analysis, the area function is an important tool to characterize the function spaces. In [7], Fefferman and Stein proved that the area function and the nontangential maximal function are equivalent in the sense of $L^{p}\left(\mathbb{R}^{n}\right)$; they also established the area function characterization of the Hardy spaces $H^{p}\left(\mathbb{R}^{n}\right)$. From then on, the Hardy space was extended to other settings. (We refer the reader to [3], [2], [8], [18], [10], and the references therein, which investigate more general

Copyright 2016 by the Tusi Mathematical Research Group.

Received Nov. 6, 2015; Accepted Jan. 6, 2016.

${ }^{*}$ Corresponding author.

2010 Mathematics Subject Classification. Primary 42B30; Secondary 35J10, 42B25.

Keywords. Hardy space, Schrödinger operator, atom, area integral, Poisson semigroup, area function. 


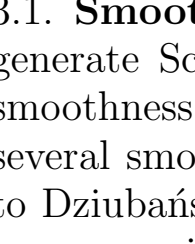

Banach J. Math. Anal. 10 (2016), no. 4, 727-749

http://dx.doi.org/10.1215/17358787-3649986

ISSN: $1735-8787$ (electronic)

http://projecteuclid.org/bjma

ANALYSIS

\title{
POISSON SEMIGROUP, AREA FUNCTION, AND THE CHARACTERIZATION OF HARDY SPACE ASSOCIATED TO DEGENERATE SCHRÖDINGER OPERATORS
}

\author{
JIZHENG HUANG, ${ }^{1}$ PENGTAO LI, ${ }^{2^{*}}$ and YU LIU ${ }^{3}$ \\ Communicated by M. A. Ragusa
}

Abstract. Let

$$
L f(x)=-\frac{1}{\omega(x)} \sum_{i, j} \partial_{i}\left(a_{i j}(\cdot) \partial_{j} f\right)(x)+V(x) f(x)
$$

be the degenerate Schrödinger operator, where $\omega$ is a weight from the Muckenhoupt class $A_{2}$ and $V$ is a nonnegative potential that belongs to a certain reverse Hölder class with respect to the measure $\omega(x) d x$. Based on some smoothness estimates of the Poisson semigroup $e^{-t \sqrt{L}}$, we introduce the area function $S_{P}^{L}$ associated with $e^{-t \sqrt{L}}$ to characterize the Hardy space associated with $L$.

\section{INTRODUCTION}

As a special class of Calderón-Zygmund singular integrals, the area function is a useful way of building bridges between real analysis and complex analysis. In harmonic analysis, the area function is an important tool to characterize the function spaces. In [7], Fefferman and Stein proved that the area function and the nontangential maximal function are equivalent in the sense of $L^{p}\left(\mathbb{R}^{n}\right)$; they also established the area function characterization of the Hardy spaces $H^{p}\left(\mathbb{R}^{n}\right)$. From then on, the Hardy space was extended to other settings. (We refer the reader to [3], [2], [8], [18], [10], and the references therein, which investigate more general

Copyright 2016 by the Tusi Mathematical Research Group.

Received Nov. 6, 2015; Accepted Jan. 6, 2016.

${ }^{*}$ Corresponding author.

2010 Mathematics Subject Classification. Primary 42B30; Secondary 35J10, 42B25.

Keywords. Hardy space, Schrödinger operator, atom, area integral, Poisson semigroup, area function. 


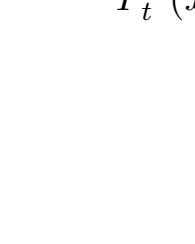

Banach J. Math. Anal. 10 (2016), no. 4, 727-749

http://dx.doi.org/10.1215/17358787-3649986

ISSN: $1735-8787$ (electronic)

http://projecteuclid.org/bjma

ANALYSIS

\title{
POISSON SEMIGROUP, AREA FUNCTION, AND THE CHARACTERIZATION OF HARDY SPACE ASSOCIATED TO DEGENERATE SCHRÖDINGER OPERATORS
}

\author{
JIZHENG HUANG, ${ }^{1}$ PENGTAO LI, ${ }^{2^{*}}$ and YU LIU ${ }^{3}$ \\ Communicated by M. A. Ragusa
}

Abstract. Let

$$
L f(x)=-\frac{1}{\omega(x)} \sum_{i, j} \partial_{i}\left(a_{i j}(\cdot) \partial_{j} f\right)(x)+V(x) f(x)
$$

be the degenerate Schrödinger operator, where $\omega$ is a weight from the Muckenhoupt class $A_{2}$ and $V$ is a nonnegative potential that belongs to a certain reverse Hölder class with respect to the measure $\omega(x) d x$. Based on some smoothness estimates of the Poisson semigroup $e^{-t \sqrt{L}}$, we introduce the area function $S_{P}^{L}$ associated with $e^{-t \sqrt{L}}$ to characterize the Hardy space associated with $L$.

\section{INTRODUCTION}

As a special class of Calderón-Zygmund singular integrals, the area function is a useful way of building bridges between real analysis and complex analysis. In harmonic analysis, the area function is an important tool to characterize the function spaces. In [7], Fefferman and Stein proved that the area function and the nontangential maximal function are equivalent in the sense of $L^{p}\left(\mathbb{R}^{n}\right)$; they also established the area function characterization of the Hardy spaces $H^{p}\left(\mathbb{R}^{n}\right)$. From then on, the Hardy space was extended to other settings. (We refer the reader to [3], [2], [8], [18], [10], and the references therein, which investigate more general

Copyright 2016 by the Tusi Mathematical Research Group.

Received Nov. 6, 2015; Accepted Jan. 6, 2016.

${ }^{*}$ Corresponding author.

2010 Mathematics Subject Classification. Primary 42B30; Secondary 35J10, 42B25.

Keywords. Hardy space, Schrödinger operator, atom, area integral, Poisson semigroup, area function. 


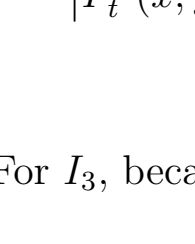

Banach J. Math. Anal. 10 (2016), no. 4, 727-749

http://dx.doi.org/10.1215/17358787-3649986

ISSN: $1735-8787$ (electronic)

http://projecteuclid.org/bjma

ANALYSIS

\title{
POISSON SEMIGROUP, AREA FUNCTION, AND THE CHARACTERIZATION OF HARDY SPACE ASSOCIATED TO DEGENERATE SCHRÖDINGER OPERATORS
}

\author{
JIZHENG HUANG, ${ }^{1}$ PENGTAO LI, ${ }^{2^{*}}$ and YU LIU ${ }^{3}$ \\ Communicated by M. A. Ragusa
}

Abstract. Let

$$
L f(x)=-\frac{1}{\omega(x)} \sum_{i, j} \partial_{i}\left(a_{i j}(\cdot) \partial_{j} f\right)(x)+V(x) f(x)
$$

be the degenerate Schrödinger operator, where $\omega$ is a weight from the Muckenhoupt class $A_{2}$ and $V$ is a nonnegative potential that belongs to a certain reverse Hölder class with respect to the measure $\omega(x) d x$. Based on some smoothness estimates of the Poisson semigroup $e^{-t \sqrt{L}}$, we introduce the area function $S_{P}^{L}$ associated with $e^{-t \sqrt{L}}$ to characterize the Hardy space associated with $L$.

\section{INTRODUCTION}

As a special class of Calderón-Zygmund singular integrals, the area function is a useful way of building bridges between real analysis and complex analysis. In harmonic analysis, the area function is an important tool to characterize the function spaces. In [7], Fefferman and Stein proved that the area function and the nontangential maximal function are equivalent in the sense of $L^{p}\left(\mathbb{R}^{n}\right)$; they also established the area function characterization of the Hardy spaces $H^{p}\left(\mathbb{R}^{n}\right)$. From then on, the Hardy space was extended to other settings. (We refer the reader to [3], [2], [8], [18], [10], and the references therein, which investigate more general

Copyright 2016 by the Tusi Mathematical Research Group.

Received Nov. 6, 2015; Accepted Jan. 6, 2016.

${ }^{*}$ Corresponding author.

2010 Mathematics Subject Classification. Primary 42B30; Secondary 35J10, 42B25.

Keywords. Hardy space, Schrödinger operator, atom, area integral, Poisson semigroup, area function. 


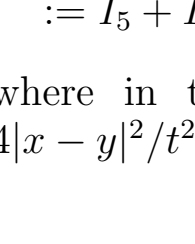

Banach J. Math. Anal. 10 (2016), no. 4, 727-749

http://dx.doi.org/10.1215/17358787-3649986

ISSN: $1735-8787$ (electronic)

http://projecteuclid.org/bjma

ANALYSIS

\title{
POISSON SEMIGROUP, AREA FUNCTION, AND THE CHARACTERIZATION OF HARDY SPACE ASSOCIATED TO DEGENERATE SCHRÖDINGER OPERATORS
}

\author{
JIZHENG HUANG, ${ }^{1}$ PENGTAO LI, ${ }^{2^{*}}$ and YU LIU ${ }^{3}$ \\ Communicated by M. A. Ragusa
}

Abstract. Let

$$
L f(x)=-\frac{1}{\omega(x)} \sum_{i, j} \partial_{i}\left(a_{i j}(\cdot) \partial_{j} f\right)(x)+V(x) f(x)
$$

be the degenerate Schrödinger operator, where $\omega$ is a weight from the Muckenhoupt class $A_{2}$ and $V$ is a nonnegative potential that belongs to a certain reverse Hölder class with respect to the measure $\omega(x) d x$. Based on some smoothness estimates of the Poisson semigroup $e^{-t \sqrt{L}}$, we introduce the area function $S_{P}^{L}$ associated with $e^{-t \sqrt{L}}$ to characterize the Hardy space associated with $L$.

\section{INTRODUCTION}

As a special class of Calderón-Zygmund singular integrals, the area function is a useful way of building bridges between real analysis and complex analysis. In harmonic analysis, the area function is an important tool to characterize the function spaces. In [7], Fefferman and Stein proved that the area function and the nontangential maximal function are equivalent in the sense of $L^{p}\left(\mathbb{R}^{n}\right)$; they also established the area function characterization of the Hardy spaces $H^{p}\left(\mathbb{R}^{n}\right)$. From then on, the Hardy space was extended to other settings. (We refer the reader to [3], [2], [8], [18], [10], and the references therein, which investigate more general

Copyright 2016 by the Tusi Mathematical Research Group.

Received Nov. 6, 2015; Accepted Jan. 6, 2016.

${ }^{*}$ Corresponding author.

2010 Mathematics Subject Classification. Primary 42B30; Secondary 35J10, 42B25.

Keywords. Hardy space, Schrödinger operator, atom, area integral, Poisson semigroup, area function. 


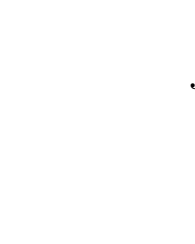

Banach J. Math. Anal. 10 (2016), no. 4, 727-749

http://dx.doi.org/10.1215/17358787-3649986

ISSN: $1735-8787$ (electronic)

http://projecteuclid.org/bjma

ANALYSIS

\title{
POISSON SEMIGROUP, AREA FUNCTION, AND THE CHARACTERIZATION OF HARDY SPACE ASSOCIATED TO DEGENERATE SCHRÖDINGER OPERATORS
}

\author{
JIZHENG HUANG, ${ }^{1}$ PENGTAO LI, ${ }^{2^{*}}$ and YU LIU ${ }^{3}$ \\ Communicated by M. A. Ragusa
}

Abstract. Let

$$
L f(x)=-\frac{1}{\omega(x)} \sum_{i, j} \partial_{i}\left(a_{i j}(\cdot) \partial_{j} f\right)(x)+V(x) f(x)
$$

be the degenerate Schrödinger operator, where $\omega$ is a weight from the Muckenhoupt class $A_{2}$ and $V$ is a nonnegative potential that belongs to a certain reverse Hölder class with respect to the measure $\omega(x) d x$. Based on some smoothness estimates of the Poisson semigroup $e^{-t \sqrt{L}}$, we introduce the area function $S_{P}^{L}$ associated with $e^{-t \sqrt{L}}$ to characterize the Hardy space associated with $L$.

\section{INTRODUCTION}

As a special class of Calderón-Zygmund singular integrals, the area function is a useful way of building bridges between real analysis and complex analysis. In harmonic analysis, the area function is an important tool to characterize the function spaces. In [7], Fefferman and Stein proved that the area function and the nontangential maximal function are equivalent in the sense of $L^{p}\left(\mathbb{R}^{n}\right)$; they also established the area function characterization of the Hardy spaces $H^{p}\left(\mathbb{R}^{n}\right)$. From then on, the Hardy space was extended to other settings. (We refer the reader to [3], [2], [8], [18], [10], and the references therein, which investigate more general

Copyright 2016 by the Tusi Mathematical Research Group.

Received Nov. 6, 2015; Accepted Jan. 6, 2016.

${ }^{*}$ Corresponding author.

2010 Mathematics Subject Classification. Primary 42B30; Secondary 35J10, 42B25.

Keywords. Hardy space, Schrödinger operator, atom, area integral, Poisson semigroup, area function. 


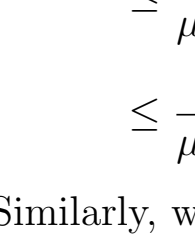

Banach J. Math. Anal. 10 (2016), no. 4, 727-749

http://dx.doi.org/10.1215/17358787-3649986

ISSN: $1735-8787$ (electronic)

http://projecteuclid.org/bjma

ANALYSIS

\title{
POISSON SEMIGROUP, AREA FUNCTION, AND THE CHARACTERIZATION OF HARDY SPACE ASSOCIATED TO DEGENERATE SCHRÖDINGER OPERATORS
}

\author{
JIZHENG HUANG, ${ }^{1}$ PENGTAO LI, ${ }^{2^{*}}$ and YU LIU ${ }^{3}$ \\ Communicated by M. A. Ragusa
}

Abstract. Let

$$
L f(x)=-\frac{1}{\omega(x)} \sum_{i, j} \partial_{i}\left(a_{i j}(\cdot) \partial_{j} f\right)(x)+V(x) f(x)
$$

be the degenerate Schrödinger operator, where $\omega$ is a weight from the Muckenhoupt class $A_{2}$ and $V$ is a nonnegative potential that belongs to a certain reverse Hölder class with respect to the measure $\omega(x) d x$. Based on some smoothness estimates of the Poisson semigroup $e^{-t \sqrt{L}}$, we introduce the area function $S_{P}^{L}$ associated with $e^{-t \sqrt{L}}$ to characterize the Hardy space associated with $L$.

\section{INTRODUCTION}

As a special class of Calderón-Zygmund singular integrals, the area function is a useful way of building bridges between real analysis and complex analysis. In harmonic analysis, the area function is an important tool to characterize the function spaces. In [7], Fefferman and Stein proved that the area function and the nontangential maximal function are equivalent in the sense of $L^{p}\left(\mathbb{R}^{n}\right)$; they also established the area function characterization of the Hardy spaces $H^{p}\left(\mathbb{R}^{n}\right)$. From then on, the Hardy space was extended to other settings. (We refer the reader to [3], [2], [8], [18], [10], and the references therein, which investigate more general

Copyright 2016 by the Tusi Mathematical Research Group.

Received Nov. 6, 2015; Accepted Jan. 6, 2016.

${ }^{*}$ Corresponding author.

2010 Mathematics Subject Classification. Primary 42B30; Secondary 35J10, 42B25.

Keywords. Hardy space, Schrödinger operator, atom, area integral, Poisson semigroup, area function. 


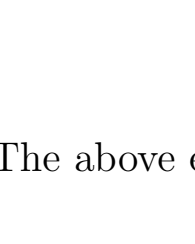

Banach J. Math. Anal. 10 (2016), no. 4, 727-749

http://dx.doi.org/10.1215/17358787-3649986

ISSN: $1735-8787$ (electronic)

http://projecteuclid.org/bjma

ANALYSIS

\title{
POISSON SEMIGROUP, AREA FUNCTION, AND THE CHARACTERIZATION OF HARDY SPACE ASSOCIATED TO DEGENERATE SCHRÖDINGER OPERATORS
}

\author{
JIZHENG HUANG, ${ }^{1}$ PENGTAO LI, ${ }^{2^{*}}$ and YU LIU ${ }^{3}$ \\ Communicated by M. A. Ragusa
}

Abstract. Let

$$
L f(x)=-\frac{1}{\omega(x)} \sum_{i, j} \partial_{i}\left(a_{i j}(\cdot) \partial_{j} f\right)(x)+V(x) f(x)
$$

be the degenerate Schrödinger operator, where $\omega$ is a weight from the Muckenhoupt class $A_{2}$ and $V$ is a nonnegative potential that belongs to a certain reverse Hölder class with respect to the measure $\omega(x) d x$. Based on some smoothness estimates of the Poisson semigroup $e^{-t \sqrt{L}}$, we introduce the area function $S_{P}^{L}$ associated with $e^{-t \sqrt{L}}$ to characterize the Hardy space associated with $L$.

\section{INTRODUCTION}

As a special class of Calderón-Zygmund singular integrals, the area function is a useful way of building bridges between real analysis and complex analysis. In harmonic analysis, the area function is an important tool to characterize the function spaces. In [7], Fefferman and Stein proved that the area function and the nontangential maximal function are equivalent in the sense of $L^{p}\left(\mathbb{R}^{n}\right)$; they also established the area function characterization of the Hardy spaces $H^{p}\left(\mathbb{R}^{n}\right)$. From then on, the Hardy space was extended to other settings. (We refer the reader to [3], [2], [8], [18], [10], and the references therein, which investigate more general

Copyright 2016 by the Tusi Mathematical Research Group.

Received Nov. 6, 2015; Accepted Jan. 6, 2016.

${ }^{*}$ Corresponding author.

2010 Mathematics Subject Classification. Primary 42B30; Secondary 35J10, 42B25.

Keywords. Hardy space, Schrödinger operator, atom, area integral, Poisson semigroup, area function. 


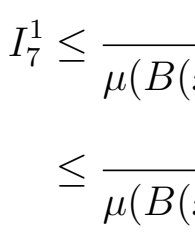

Banach J. Math. Anal. 10 (2016), no. 4, 727-749

http://dx.doi.org/10.1215/17358787-3649986

ISSN: $1735-8787$ (electronic)

http://projecteuclid.org/bjma

ANALYSIS

\title{
POISSON SEMIGROUP, AREA FUNCTION, AND THE CHARACTERIZATION OF HARDY SPACE ASSOCIATED TO DEGENERATE SCHRÖDINGER OPERATORS
}

\author{
JIZHENG HUANG, ${ }^{1}$ PENGTAO LI, ${ }^{2^{*}}$ and YU LIU ${ }^{3}$ \\ Communicated by M. A. Ragusa
}

Abstract. Let

$$
L f(x)=-\frac{1}{\omega(x)} \sum_{i, j} \partial_{i}\left(a_{i j}(\cdot) \partial_{j} f\right)(x)+V(x) f(x)
$$

be the degenerate Schrödinger operator, where $\omega$ is a weight from the Muckenhoupt class $A_{2}$ and $V$ is a nonnegative potential that belongs to a certain reverse Hölder class with respect to the measure $\omega(x) d x$. Based on some smoothness estimates of the Poisson semigroup $e^{-t \sqrt{L}}$, we introduce the area function $S_{P}^{L}$ associated with $e^{-t \sqrt{L}}$ to characterize the Hardy space associated with $L$.

\section{INTRODUCTION}

As a special class of Calderón-Zygmund singular integrals, the area function is a useful way of building bridges between real analysis and complex analysis. In harmonic analysis, the area function is an important tool to characterize the function spaces. In [7], Fefferman and Stein proved that the area function and the nontangential maximal function are equivalent in the sense of $L^{p}\left(\mathbb{R}^{n}\right)$; they also established the area function characterization of the Hardy spaces $H^{p}\left(\mathbb{R}^{n}\right)$. From then on, the Hardy space was extended to other settings. (We refer the reader to [3], [2], [8], [18], [10], and the references therein, which investigate more general

Copyright 2016 by the Tusi Mathematical Research Group.

Received Nov. 6, 2015; Accepted Jan. 6, 2016.

${ }^{*}$ Corresponding author.

2010 Mathematics Subject Classification. Primary 42B30; Secondary 35J10, 42B25.

Keywords. Hardy space, Schrödinger operator, atom, area integral, Poisson semigroup, area function. 


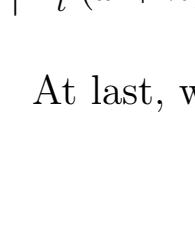

Banach J. Math. Anal. 10 (2016), no. 4, 727-749

http://dx.doi.org/10.1215/17358787-3649986

ISSN: $1735-8787$ (electronic)

http://projecteuclid.org/bjma

ANALYSIS

\title{
POISSON SEMIGROUP, AREA FUNCTION, AND THE CHARACTERIZATION OF HARDY SPACE ASSOCIATED TO DEGENERATE SCHRÖDINGER OPERATORS
}

\author{
JIZHENG HUANG, ${ }^{1}$ PENGTAO LI, ${ }^{2^{*}}$ and YU LIU ${ }^{3}$ \\ Communicated by M. A. Ragusa
}

Abstract. Let

$$
L f(x)=-\frac{1}{\omega(x)} \sum_{i, j} \partial_{i}\left(a_{i j}(\cdot) \partial_{j} f\right)(x)+V(x) f(x)
$$

be the degenerate Schrödinger operator, where $\omega$ is a weight from the Muckenhoupt class $A_{2}$ and $V$ is a nonnegative potential that belongs to a certain reverse Hölder class with respect to the measure $\omega(x) d x$. Based on some smoothness estimates of the Poisson semigroup $e^{-t \sqrt{L}}$, we introduce the area function $S_{P}^{L}$ associated with $e^{-t \sqrt{L}}$ to characterize the Hardy space associated with $L$.

\section{INTRODUCTION}

As a special class of Calderón-Zygmund singular integrals, the area function is a useful way of building bridges between real analysis and complex analysis. In harmonic analysis, the area function is an important tool to characterize the function spaces. In [7], Fefferman and Stein proved that the area function and the nontangential maximal function are equivalent in the sense of $L^{p}\left(\mathbb{R}^{n}\right)$; they also established the area function characterization of the Hardy spaces $H^{p}\left(\mathbb{R}^{n}\right)$. From then on, the Hardy space was extended to other settings. (We refer the reader to [3], [2], [8], [18], [10], and the references therein, which investigate more general

Copyright 2016 by the Tusi Mathematical Research Group.

Received Nov. 6, 2015; Accepted Jan. 6, 2016.

${ }^{*}$ Corresponding author.

2010 Mathematics Subject Classification. Primary 42B30; Secondary 35J10, 42B25.

Keywords. Hardy space, Schrödinger operator, atom, area integral, Poisson semigroup, area function. 


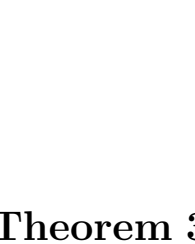

Banach J. Math. Anal. 10 (2016), no. 4, 727-749

http://dx.doi.org/10.1215/17358787-3649986

ISSN: $1735-8787$ (electronic)

http://projecteuclid.org/bjma

ANALYSIS

\title{
POISSON SEMIGROUP, AREA FUNCTION, AND THE CHARACTERIZATION OF HARDY SPACE ASSOCIATED TO DEGENERATE SCHRÖDINGER OPERATORS
}

\author{
JIZHENG HUANG, ${ }^{1}$ PENGTAO LI, ${ }^{2^{*}}$ and YU LIU ${ }^{3}$ \\ Communicated by M. A. Ragusa
}

Abstract. Let

$$
L f(x)=-\frac{1}{\omega(x)} \sum_{i, j} \partial_{i}\left(a_{i j}(\cdot) \partial_{j} f\right)(x)+V(x) f(x)
$$

be the degenerate Schrödinger operator, where $\omega$ is a weight from the Muckenhoupt class $A_{2}$ and $V$ is a nonnegative potential that belongs to a certain reverse Hölder class with respect to the measure $\omega(x) d x$. Based on some smoothness estimates of the Poisson semigroup $e^{-t \sqrt{L}}$, we introduce the area function $S_{P}^{L}$ associated with $e^{-t \sqrt{L}}$ to characterize the Hardy space associated with $L$.

\section{INTRODUCTION}

As a special class of Calderón-Zygmund singular integrals, the area function is a useful way of building bridges between real analysis and complex analysis. In harmonic analysis, the area function is an important tool to characterize the function spaces. In [7], Fefferman and Stein proved that the area function and the nontangential maximal function are equivalent in the sense of $L^{p}\left(\mathbb{R}^{n}\right)$; they also established the area function characterization of the Hardy spaces $H^{p}\left(\mathbb{R}^{n}\right)$. From then on, the Hardy space was extended to other settings. (We refer the reader to [3], [2], [8], [18], [10], and the references therein, which investigate more general

Copyright 2016 by the Tusi Mathematical Research Group.

Received Nov. 6, 2015; Accepted Jan. 6, 2016.

${ }^{*}$ Corresponding author.

2010 Mathematics Subject Classification. Primary 42B30; Secondary 35J10, 42B25.

Keywords. Hardy space, Schrödinger operator, atom, area integral, Poisson semigroup, area function. 


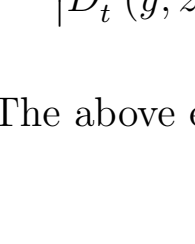

Banach J. Math. Anal. 10 (2016), no. 4, 727-749

http://dx.doi.org/10.1215/17358787-3649986

ISSN: $1735-8787$ (electronic)

http://projecteuclid.org/bjma

ANALYSIS

\title{
POISSON SEMIGROUP, AREA FUNCTION, AND THE CHARACTERIZATION OF HARDY SPACE ASSOCIATED TO DEGENERATE SCHRÖDINGER OPERATORS
}

\author{
JIZHENG HUANG, ${ }^{1}$ PENGTAO LI, ${ }^{2^{*}}$ and YU LIU ${ }^{3}$ \\ Communicated by M. A. Ragusa
}

Abstract. Let

$$
L f(x)=-\frac{1}{\omega(x)} \sum_{i, j} \partial_{i}\left(a_{i j}(\cdot) \partial_{j} f\right)(x)+V(x) f(x)
$$

be the degenerate Schrödinger operator, where $\omega$ is a weight from the Muckenhoupt class $A_{2}$ and $V$ is a nonnegative potential that belongs to a certain reverse Hölder class with respect to the measure $\omega(x) d x$. Based on some smoothness estimates of the Poisson semigroup $e^{-t \sqrt{L}}$, we introduce the area function $S_{P}^{L}$ associated with $e^{-t \sqrt{L}}$ to characterize the Hardy space associated with $L$.

\section{INTRODUCTION}

As a special class of Calderón-Zygmund singular integrals, the area function is a useful way of building bridges between real analysis and complex analysis. In harmonic analysis, the area function is an important tool to characterize the function spaces. In [7], Fefferman and Stein proved that the area function and the nontangential maximal function are equivalent in the sense of $L^{p}\left(\mathbb{R}^{n}\right)$; they also established the area function characterization of the Hardy spaces $H^{p}\left(\mathbb{R}^{n}\right)$. From then on, the Hardy space was extended to other settings. (We refer the reader to [3], [2], [8], [18], [10], and the references therein, which investigate more general

Copyright 2016 by the Tusi Mathematical Research Group.

Received Nov. 6, 2015; Accepted Jan. 6, 2016.

${ }^{*}$ Corresponding author.

2010 Mathematics Subject Classification. Primary 42B30; Secondary 35J10, 42B25.

Keywords. Hardy space, Schrödinger operator, atom, area integral, Poisson semigroup, area function. 


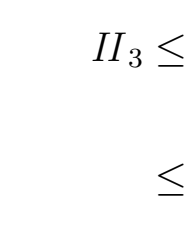

Banach J. Math. Anal. 10 (2016), no. 4, 727-749

http://dx.doi.org/10.1215/17358787-3649986

ISSN: $1735-8787$ (electronic)

http://projecteuclid.org/bjma

ANALYSIS

\title{
POISSON SEMIGROUP, AREA FUNCTION, AND THE CHARACTERIZATION OF HARDY SPACE ASSOCIATED TO DEGENERATE SCHRÖDINGER OPERATORS
}

\author{
JIZHENG HUANG, ${ }^{1}$ PENGTAO LI, ${ }^{2^{*}}$ and YU LIU ${ }^{3}$ \\ Communicated by M. A. Ragusa
}

Abstract. Let

$$
L f(x)=-\frac{1}{\omega(x)} \sum_{i, j} \partial_{i}\left(a_{i j}(\cdot) \partial_{j} f\right)(x)+V(x) f(x)
$$

be the degenerate Schrödinger operator, where $\omega$ is a weight from the Muckenhoupt class $A_{2}$ and $V$ is a nonnegative potential that belongs to a certain reverse Hölder class with respect to the measure $\omega(x) d x$. Based on some smoothness estimates of the Poisson semigroup $e^{-t \sqrt{L}}$, we introduce the area function $S_{P}^{L}$ associated with $e^{-t \sqrt{L}}$ to characterize the Hardy space associated with $L$.

\section{INTRODUCTION}

As a special class of Calderón-Zygmund singular integrals, the area function is a useful way of building bridges between real analysis and complex analysis. In harmonic analysis, the area function is an important tool to characterize the function spaces. In [7], Fefferman and Stein proved that the area function and the nontangential maximal function are equivalent in the sense of $L^{p}\left(\mathbb{R}^{n}\right)$; they also established the area function characterization of the Hardy spaces $H^{p}\left(\mathbb{R}^{n}\right)$. From then on, the Hardy space was extended to other settings. (We refer the reader to [3], [2], [8], [18], [10], and the references therein, which investigate more general

Copyright 2016 by the Tusi Mathematical Research Group.

Received Nov. 6, 2015; Accepted Jan. 6, 2016.

${ }^{*}$ Corresponding author.

2010 Mathematics Subject Classification. Primary 42B30; Secondary 35J10, 42B25.

Keywords. Hardy space, Schrödinger operator, atom, area integral, Poisson semigroup, area function. 


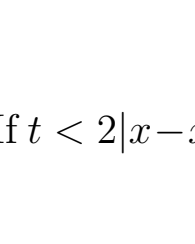

Banach J. Math. Anal. 10 (2016), no. 4, 727-749

http://dx.doi.org/10.1215/17358787-3649986

ISSN: $1735-8787$ (electronic)

http://projecteuclid.org/bjma

ANALYSIS

\title{
POISSON SEMIGROUP, AREA FUNCTION, AND THE CHARACTERIZATION OF HARDY SPACE ASSOCIATED TO DEGENERATE SCHRÖDINGER OPERATORS
}

\author{
JIZHENG HUANG, ${ }^{1}$ PENGTAO LI, ${ }^{2^{*}}$ and YU LIU ${ }^{3}$ \\ Communicated by M. A. Ragusa
}

Abstract. Let

$$
L f(x)=-\frac{1}{\omega(x)} \sum_{i, j} \partial_{i}\left(a_{i j}(\cdot) \partial_{j} f\right)(x)+V(x) f(x)
$$

be the degenerate Schrödinger operator, where $\omega$ is a weight from the Muckenhoupt class $A_{2}$ and $V$ is a nonnegative potential that belongs to a certain reverse Hölder class with respect to the measure $\omega(x) d x$. Based on some smoothness estimates of the Poisson semigroup $e^{-t \sqrt{L}}$, we introduce the area function $S_{P}^{L}$ associated with $e^{-t \sqrt{L}}$ to characterize the Hardy space associated with $L$.

\section{INTRODUCTION}

As a special class of Calderón-Zygmund singular integrals, the area function is a useful way of building bridges between real analysis and complex analysis. In harmonic analysis, the area function is an important tool to characterize the function spaces. In [7], Fefferman and Stein proved that the area function and the nontangential maximal function are equivalent in the sense of $L^{p}\left(\mathbb{R}^{n}\right)$; they also established the area function characterization of the Hardy spaces $H^{p}\left(\mathbb{R}^{n}\right)$. From then on, the Hardy space was extended to other settings. (We refer the reader to [3], [2], [8], [18], [10], and the references therein, which investigate more general

Copyright 2016 by the Tusi Mathematical Research Group.

Received Nov. 6, 2015; Accepted Jan. 6, 2016.

${ }^{*}$ Corresponding author.

2010 Mathematics Subject Classification. Primary 42B30; Secondary 35J10, 42B25.

Keywords. Hardy space, Schrödinger operator, atom, area integral, Poisson semigroup, area function. 


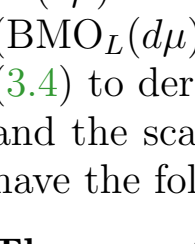

Banach J. Math. Anal. 10 (2016), no. 4, 727-749

http://dx.doi.org/10.1215/17358787-3649986

ISSN: $1735-8787$ (electronic)

http://projecteuclid.org/bjma

ANALYSIS

\title{
POISSON SEMIGROUP, AREA FUNCTION, AND THE CHARACTERIZATION OF HARDY SPACE ASSOCIATED TO DEGENERATE SCHRÖDINGER OPERATORS
}

\author{
JIZHENG HUANG, ${ }^{1}$ PENGTAO LI, ${ }^{2^{*}}$ and YU LIU ${ }^{3}$ \\ Communicated by M. A. Ragusa
}

Abstract. Let

$$
L f(x)=-\frac{1}{\omega(x)} \sum_{i, j} \partial_{i}\left(a_{i j}(\cdot) \partial_{j} f\right)(x)+V(x) f(x)
$$

be the degenerate Schrödinger operator, where $\omega$ is a weight from the Muckenhoupt class $A_{2}$ and $V$ is a nonnegative potential that belongs to a certain reverse Hölder class with respect to the measure $\omega(x) d x$. Based on some smoothness estimates of the Poisson semigroup $e^{-t \sqrt{L}}$, we introduce the area function $S_{P}^{L}$ associated with $e^{-t \sqrt{L}}$ to characterize the Hardy space associated with $L$.

\section{INTRODUCTION}

As a special class of Calderón-Zygmund singular integrals, the area function is a useful way of building bridges between real analysis and complex analysis. In harmonic analysis, the area function is an important tool to characterize the function spaces. In [7], Fefferman and Stein proved that the area function and the nontangential maximal function are equivalent in the sense of $L^{p}\left(\mathbb{R}^{n}\right)$; they also established the area function characterization of the Hardy spaces $H^{p}\left(\mathbb{R}^{n}\right)$. From then on, the Hardy space was extended to other settings. (We refer the reader to [3], [2], [8], [18], [10], and the references therein, which investigate more general

Copyright 2016 by the Tusi Mathematical Research Group.

Received Nov. 6, 2015; Accepted Jan. 6, 2016.

${ }^{*}$ Corresponding author.

2010 Mathematics Subject Classification. Primary 42B30; Secondary 35J10, 42B25.

Keywords. Hardy space, Schrödinger operator, atom, area integral, Poisson semigroup, area function. 


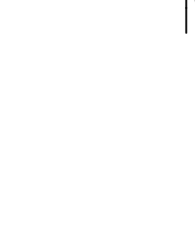

Banach J. Math. Anal. 10 (2016), no. 4, 727-749

http://dx.doi.org/10.1215/17358787-3649986

ISSN: $1735-8787$ (electronic)

http://projecteuclid.org/bjma

ANALYSIS

\title{
POISSON SEMIGROUP, AREA FUNCTION, AND THE CHARACTERIZATION OF HARDY SPACE ASSOCIATED TO DEGENERATE SCHRÖDINGER OPERATORS
}

\author{
JIZHENG HUANG, ${ }^{1}$ PENGTAO LI, ${ }^{2^{*}}$ and YU LIU ${ }^{3}$ \\ Communicated by M. A. Ragusa
}

Abstract. Let

$$
L f(x)=-\frac{1}{\omega(x)} \sum_{i, j} \partial_{i}\left(a_{i j}(\cdot) \partial_{j} f\right)(x)+V(x) f(x)
$$

be the degenerate Schrödinger operator, where $\omega$ is a weight from the Muckenhoupt class $A_{2}$ and $V$ is a nonnegative potential that belongs to a certain reverse Hölder class with respect to the measure $\omega(x) d x$. Based on some smoothness estimates of the Poisson semigroup $e^{-t \sqrt{L}}$, we introduce the area function $S_{P}^{L}$ associated with $e^{-t \sqrt{L}}$ to characterize the Hardy space associated with $L$.

\section{INTRODUCTION}

As a special class of Calderón-Zygmund singular integrals, the area function is a useful way of building bridges between real analysis and complex analysis. In harmonic analysis, the area function is an important tool to characterize the function spaces. In [7], Fefferman and Stein proved that the area function and the nontangential maximal function are equivalent in the sense of $L^{p}\left(\mathbb{R}^{n}\right)$; they also established the area function characterization of the Hardy spaces $H^{p}\left(\mathbb{R}^{n}\right)$. From then on, the Hardy space was extended to other settings. (We refer the reader to [3], [2], [8], [18], [10], and the references therein, which investigate more general

Copyright 2016 by the Tusi Mathematical Research Group.

Received Nov. 6, 2015; Accepted Jan. 6, 2016.

${ }^{*}$ Corresponding author.

2010 Mathematics Subject Classification. Primary 42B30; Secondary 35J10, 42B25.

Keywords. Hardy space, Schrödinger operator, atom, area integral, Poisson semigroup, area function. 


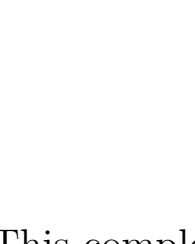

Banach J. Math. Anal. 10 (2016), no. 4, 727-749

http://dx.doi.org/10.1215/17358787-3649986

ISSN: $1735-8787$ (electronic)

http://projecteuclid.org/bjma

ANALYSIS

\title{
POISSON SEMIGROUP, AREA FUNCTION, AND THE CHARACTERIZATION OF HARDY SPACE ASSOCIATED TO DEGENERATE SCHRÖDINGER OPERATORS
}

\author{
JIZHENG HUANG, ${ }^{1}$ PENGTAO LI, ${ }^{2^{*}}$ and YU LIU ${ }^{3}$ \\ Communicated by M. A. Ragusa
}

Abstract. Let

$$
L f(x)=-\frac{1}{\omega(x)} \sum_{i, j} \partial_{i}\left(a_{i j}(\cdot) \partial_{j} f\right)(x)+V(x) f(x)
$$

be the degenerate Schrödinger operator, where $\omega$ is a weight from the Muckenhoupt class $A_{2}$ and $V$ is a nonnegative potential that belongs to a certain reverse Hölder class with respect to the measure $\omega(x) d x$. Based on some smoothness estimates of the Poisson semigroup $e^{-t \sqrt{L}}$, we introduce the area function $S_{P}^{L}$ associated with $e^{-t \sqrt{L}}$ to characterize the Hardy space associated with $L$.

\section{INTRODUCTION}

As a special class of Calderón-Zygmund singular integrals, the area function is a useful way of building bridges between real analysis and complex analysis. In harmonic analysis, the area function is an important tool to characterize the function spaces. In [7], Fefferman and Stein proved that the area function and the nontangential maximal function are equivalent in the sense of $L^{p}\left(\mathbb{R}^{n}\right)$; they also established the area function characterization of the Hardy spaces $H^{p}\left(\mathbb{R}^{n}\right)$. From then on, the Hardy space was extended to other settings. (We refer the reader to [3], [2], [8], [18], [10], and the references therein, which investigate more general

Copyright 2016 by the Tusi Mathematical Research Group.

Received Nov. 6, 2015; Accepted Jan. 6, 2016.

${ }^{*}$ Corresponding author.

2010 Mathematics Subject Classification. Primary 42B30; Secondary 35J10, 42B25.

Keywords. Hardy space, Schrödinger operator, atom, area integral, Poisson semigroup, area function. 


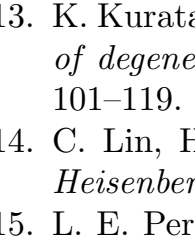

Banach J. Math. Anal. 10 (2016), no. 4, 727-749

http://dx.doi.org/10.1215/17358787-3649986

ISSN: $1735-8787$ (electronic)

http://projecteuclid.org/bjma

ANALYSIS

\title{
POISSON SEMIGROUP, AREA FUNCTION, AND THE CHARACTERIZATION OF HARDY SPACE ASSOCIATED TO DEGENERATE SCHRÖDINGER OPERATORS
}

\author{
JIZHENG HUANG, ${ }^{1}$ PENGTAO LI, ${ }^{2^{*}}$ and YU LIU ${ }^{3}$ \\ Communicated by M. A. Ragusa
}

Abstract. Let

$$
L f(x)=-\frac{1}{\omega(x)} \sum_{i, j} \partial_{i}\left(a_{i j}(\cdot) \partial_{j} f\right)(x)+V(x) f(x)
$$

be the degenerate Schrödinger operator, where $\omega$ is a weight from the Muckenhoupt class $A_{2}$ and $V$ is a nonnegative potential that belongs to a certain reverse Hölder class with respect to the measure $\omega(x) d x$. Based on some smoothness estimates of the Poisson semigroup $e^{-t \sqrt{L}}$, we introduce the area function $S_{P}^{L}$ associated with $e^{-t \sqrt{L}}$ to characterize the Hardy space associated with $L$.

\section{INTRODUCTION}

As a special class of Calderón-Zygmund singular integrals, the area function is a useful way of building bridges between real analysis and complex analysis. In harmonic analysis, the area function is an important tool to characterize the function spaces. In [7], Fefferman and Stein proved that the area function and the nontangential maximal function are equivalent in the sense of $L^{p}\left(\mathbb{R}^{n}\right)$; they also established the area function characterization of the Hardy spaces $H^{p}\left(\mathbb{R}^{n}\right)$. From then on, the Hardy space was extended to other settings. (We refer the reader to [3], [2], [8], [18], [10], and the references therein, which investigate more general

Copyright 2016 by the Tusi Mathematical Research Group.

Received Nov. 6, 2015; Accepted Jan. 6, 2016.

${ }^{*}$ Corresponding author.

2010 Mathematics Subject Classification. Primary 42B30; Secondary 35J10, 42B25.

Keywords. Hardy space, Schrödinger operator, atom, area integral, Poisson semigroup, area function. 